\title{
Les conditions de référence de la directive cadre européenne sur l'eau face à la dynamique des hydrosystèmes et des usages
}

\author{
Gabrielle Bouleau ${ }^{1}$, Didier Pont ${ }^{2}$ \\ ${ }^{1}$ Socio-politiste, IRSTEA, UR ADBX Aménités et dynamiques des espaces ruraux, 33612 Gazinet Cestas, France \\ 2 Écologue des milieux aquatiques, IRSTEA, UR Hydrosystèmes et bioprocédés, 92761 Antony cedex, France
}

\begin{abstract}
À l'interface entre politiques de l'environnement et connaissances scientifiques écologiques, la directive cadre européenne sur l'eau (DCE) est un cas exemplaire sous de multiples aspects : une inscription claire dans le New Public Management (NPM) qui envahit nos appareils d'État, une traduction du «bon état écologique » en termes d'indicateurs mesurant l'éloignement à des conditions de référence par ailleurs objets de débat dans la recherche écologique, une opportunité pour définir et appliquer des itinéraires de restauration. Le travail à deux voix d'une sociologue et d'un écologue produit ici une analyse fine des conditions d'émergence et de mise en œuvre de la DCE tout en identifiant les contours de futurs débats (restauration, appropriation citoyenne) au-delà même du terme prévu de la directive (en 2027).
\end{abstract}

La Rédaction

\section{Mots-clés :}

environnement ; changement climatique ; hydrobiologie ; indicateurs ; New Public Management
Résumé - La directive cadre européenne sur l'eau (DCE) ${ }^{1}$ impose une nouvelle forme de gestion des milieux aquatiques et la notion de conditions de référence est un point-clé pour la mise en œuvre de l'évaluation (mesurée comme un écart à cette référence) et pour définir les objectifs de restauration. Cependant, de nombreuses critiques se sont élevées contre ce concept. Le contexte de «changement global $^{2} \gg$ ne fait que renforcer cette tendance. Dans cet article, coécrit par un écologue et une sociopolitiste, nous revenons sur la construction sociale et scientifique de cette notion, des indicateurs qui en découlent et de leur agrégation dans la DCE. En nous focalisant sur son application aux cours d'eau, nous expliquons son origine, son succès, ses limites et les questions que pose son institutionnalisation. Cela nous conduit à nous interroger sur l'avenir de cette politique, en particulier du point de vue de la restauration.

\section{Keywords:}

environment; climate change; hydrobiology; indicators;

New Public Management

\begin{abstract}
The reference conditions in the EU Water Framework Directive confronted with the dynamics of hydrosystems and their uses. The reference conditions are a key concept of the European Water Framework Directive (WFD). This directive holds that the ecological status of a particular water body shall be assessed by quantifying the deviation from the typical reference conditions which refer to a stable state of an ecosystem in the absence of significant human disturbance. This concept is subject to criticism from several authors, all the more since global change will deeply modify the functioning of aquatic ecosystems and their associated biocenosis, whatever the impacts of human activities at local scale. In this paper, an ecologist and a sociologist reconsider the social and scientific construction of the reference conditions concept and associated rules ("one out - all out"). We explain its origin, success and limits, as well as the questions it raises. We point out aspects not considered in this concept and why it challenges the WFD implementation and future management of rivers.
\end{abstract}

\footnotetext{
Auteur correspondant : G. Bouleau, gabrielle.bouleau@irstea.fr

1 La directive cadre (2000/60/CE), adoptée le 23 octobre 2000 par le Parlement européen et le Conseil de l’Europe, établit « un cadre pour une politique communautaire dans le domaine de l'eau » (L 327 du 22/12/2000, p. 0001-0073). Elle a été transposée en droit français par la loi 2004.338 du 21 avril 2004.

2 Les auteurs admettent avec le Groupe d'experts intergouvernemental sur l'évolution du climat (Giec) la forte probabilité d'un changement climatique important dont on ne sait pas quelle sera exactement la déclinaison dans chaque région. $S^{\prime} y$ ajoutent des changements rapides et déjà constatés en matière de démographie et d'occupation du sol, et des modifications des régimes hydrologiques.
} 


\section{Introduction}

La DCE a pour objectif de préserver et d'améliorer si besoin l'état écologique des systèmes aquatiques afin d'en promouvoir un usage durable et de garantir l'approvisionnement en eau. Dans tous les cas, les États membres doivent réduire l'usage des substances dangereuses et veiller à ce que la tarification de l'eau incite aux économies. Ils doivent définir des objectifs de qualité pour chaque masse d'eau (unité hydrographique ou hydrogéologique cohérente), effectuer une évaluation et en déduire les mesures de restauration les plus appropriées.

L'évaluation de l'état écologique est tout à fait novatrice en matière de politique de qualité de l'eau, les directives antérieures n'ayant jamais imposé que des normes en matière de chimie de l'eau. Mais l'intégration de la biologie pose justement des questions sur la manière de quantifier et de juger la biodiversité dans les écosystèmes aquatiques, ainsi que sur la prise en compte (ou non) de l'évolution temporelle des systèmes.

Dans cet article $^{3}$, nous proposons de croiser les regards du sociologue et de l'écologue sur les concepts et les outils associés à cette directive pour pointer les enjeux de son application sur les cours d'eau dans un contexte de changement global. Nous nous intéressons en particulier à la traduction en termes techniques par les experts écologues et la communauté scientifique associée des notions de bon état et de conditions de référence, au travers de la mise en place de la DCE durant les vingt dernières années: de la première version du texte produite en 1994 à la fin du processus d'intercalibration européenne des bio-indicateurs en avril 2012. L'intérêt de cet échange interdisciplinaire est de mettre en évidence des convergences non intentionnelles entre différentes théories écologiques, politiques et managériales, d'expliquer comment leur combinaison a pu faire sens pour certains acteurs à certains moments et d'éclairer ainsi différentes notions de la DCE qui sont difficilement applicables dans un contexte de changement global. Cet échange nous permet aussi de pointer les avancées pragmatiques permises par ce texte et de proposer des pistes de réflexion pour le futur.

\footnotetext{
3 Cet article est issu des recherches menées dans le cadre du projet Makara (réponses sociales au changement de qualité des eaux de surface : $\mathrm{XIX}^{\mathrm{e}}$ et $\mathrm{X} \mathrm{X}^{\mathrm{e}}$ siècles) financé par l'ANR (ANR12-SENV-009), du séminaire Chiffre (Construction historique des indicateurs de faune et flore - dans l'eau et la forêt - et représentations de l'environnement) financé par l'Irstea (APR Indicateurs écologiques) et du projet européen Reform (European Program FP7-ENV-2011 No. 282656, "Restoring Rivers for Effective Catchment Management").
}

\section{Des indicateurs qui répondent à une demande des financeurs de l'environnement}

La DCE propose un cadre qui permet d'évaluer les politiques de l'eau. Des objectifs de qualité doivent être fixés en relation avec des conditions de référence, les actions pour les atteindre devront être comparées en matière de coûts et d'efficacité, une information régulière sur la qualité des eaux sera produite et rendue publique. La DCE rend ainsi les politiques de l'eau davantage comptables des deniers publics investis. Elle prévoit que cette redevabilité s'exprime sous forme d'indicateurs. En cela, cette réglementation européenne répond aux préoccupations des financeurs des politiques de l'eau.

Cependant ces financeurs n'ont pas toujours demandé d'évaluation par indicateurs. L'accent mis sur la performance quantifiable est une évolution récente qui caractérise la doctrine managériale du New Public Management et qui n'est pas spécifique au domaine de l'eau. Cette doctrine, connue pour ses applications initiales par le président américain Reagan et le Premier ministre britannique Thatcher, est depuis promue par l'OCDE (1995) et la Banque mondiale (Perrin, 2006). Elle vise à développer dans l'administration les méthodes de rationalisation des entreprises privées. Son succès s'explique parce qu'elle accompagne l'essor de l'industrie de l'audit et du conseil, en promettant aux financeurs et au pouvoir politique un contrôle plus facile (Bezes, 2005), même si la complexité de la définition des missions de service public se réduit mal à quelques chiffres. L'obligation de résultat inscrite dans la DCE est la traduction à l'échelle européenne et dans le domaine de l'environnement d'un phénomène plus large.

Il faut noter que dans les faits cet impératif d'évaluation ne s'applique pas à tous les secteurs ni à tous les échelons de l'action publique de la même manière. Les politiques européennes sont plus systématiquement évaluées que les politiques nationales. Cela s'explique $\mathrm{du}$ fait que l'essentiel des pouvoirs politiques de l'Union européenne est de nature juridique (Majone, 1996). L'évaluation est donc un contre-pouvoir vis-à-vis des États membres, avec la possibilité d'un recours contentieux. Avec la stratégie de Lisbonne de mars 2000, l'Union européenne a explicitement adopté un mode de gouvernement par les indicateurs (Dehousse, 2004). Dans le domaine de l'eau, cette évolution est également voulue par le Parlement européen et notamment sa commission de l'environnement très motivée sur la question de l'eau et très critique sur l'application de la directive sur les eaux résiduaires urbaines et sur celle concernant les nitrates (Collins et Earnshaw, 1992 ; Richardson, 1994). On sait que le Parlement a joué un rôle très important dans l'adoption de la DCE du fait de l'entrée en 


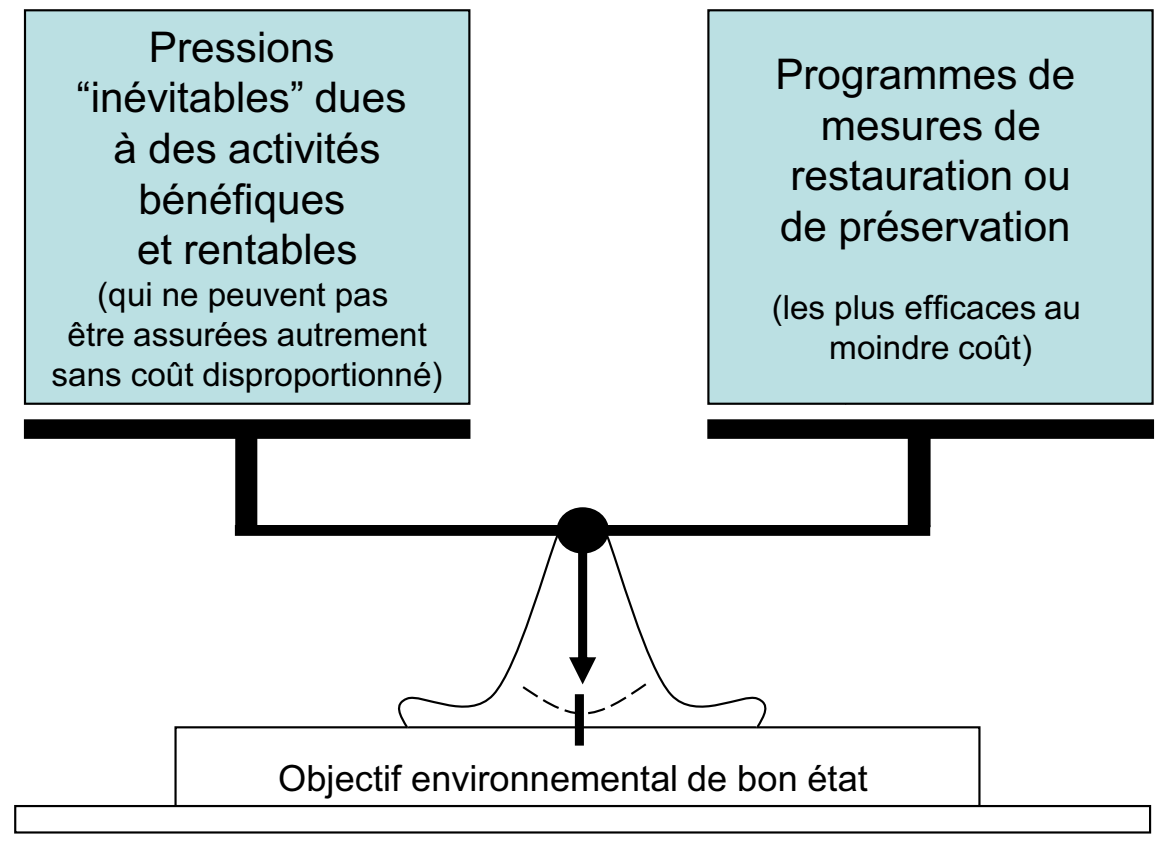

Fig. 1. Illustration de la logique compensatrice de la DCE.

vigueur du traité d'Amsterdam pendant sa négociation en 1999, alors que le conseil des ministres avait jusqu'alors le droit de veto dans le domaine de l'environnement. La contribution du Parlement a notamment porté sur le caractère contraignant des objectifs et la réduction des échéances pour les atteindre (Kaika et Page, 2003).

Selon le New Public Management, ce n'est plus aux États de prévoir et de justifier les actions à mener, mais ils doivent veiller à ce que les actions menées par d'autres soient les plus efficaces au moindre coût. Dans le domaine de l'environnement, l'OCDE a développé depuis 1993 un cadre conceptuel (pressure, state, response) permettant de comparer les actions (responses) ayant pour objectif de réduire les mêmes impacts pour atteindre un état souhaité (OECD, 1993 ; OCDE, 1994). Ce cadre a ensuite été modifié (driving force, pressure, state, impact, response ou DPSIR) par l'Agence européenne de l'environnement, et utilisé pour faire un état des eaux en Europe et des politiques de qualité de l'eau (EEA, 1995). Comme l'analyse coût-bénéfice, le modèle DPSIR est fondé sur une logique de compensation (Fernandez et al., 2011). Il s'agit de compenser des pressions induites par des forçages socioéconomiques en apportant des réponses politiques ou économiques pour éviter que l'état de l'environnement ne se dégrade en produisant des impacts sur les activités humaines. Pressions et réponses sont rendues commensurables par des indicateurs. La logique de ce modèle sous-tend toute la DCE, comme en témoignent les occurrences des termes impact (67), pressures (26), status (242) et measures (126) employés dans ce sens dans la version anglaise ${ }^{4}$. Le rôle dévolu à l'État est celui d'impulser les conditions de mise en œuvre d'une gouvernance adéquate dont la performance sera contrôlée par la Commission européenne.

Mis à part quelques restrictions sur lesquelles nous reviendrons dans la quatrième partie, la directive impose des programmes de mesures les plus efficaces au moindre coût pour compenser des pressions jugées « inévitables », parce qu'elles sont bénéfiques pour la société et rentables (Fig. 1). Ce modèle de compensation vise à restaurer l'objectif environnemental de chaque masse d'eau qui est considéré comme un point d'équilibre. Les actions de restauration sont supposées être comparables en matière d'efficacité et de coût pour permettre d'optimiser des programmes. Dans la lignée de la stratégie de Lisbonne, il est important pour l'Union européenne que la manière de définir ce point d'équilibre soit la même partout. La logique imposait donc de trouver un moyen de rendre toutes les eaux comparables à une situation qui fasse référence ou qui ne soit pas très éloignée de celle-ci. Justifier cette référence par l'existence d'un état «naturel » est une rhétorique très classique pour les institutions (Douglas, 1999).

\footnotetext{
4 Le mot state étant réservé à l'expression «Member State » (État membre) et le mot response aux éléments du rapport de la Commission en réponse aux rapports des États membres, pour parler de l'état environnemental des eaux la directive utilise le mot status et le mot measures pour les actions menées par les États pour restaurer les milieux. Pour évoquer les mesures métrologiques, la directive utilise le mot measurements.
} 
Cette lecture sociohistorique des politiques publiques de l'environnement nous permet ainsi de comprendre la notion de conditions de référence dans la DCE comme le concept qui était indispensable pour rendre les politiques de l'eau plus compatibles avec les logiques d'audit qui prévalent tant au niveau de l'OCDE que de l'Union européenne. Cette construction intellectuelle au niveau européen a rencontré l'enthousiasme de plusieurs acteurs nationaux en charge de la gestion des milieux aquatiques, qui sont depuis longtemps à la recherche d'un argumentaire économique justifiant la préservation des milieux et permettant d'établir des priorités : les agences de l'eau, le Conseil supérieur de la pêche, puis l'Office national de l'eau et des milieux aquatiques (Onema) créé postérieurement à la DCE. Cependant cette construction conceptuelle n'aurait probablement pas résisté aux critiques si elle n'avait trouvé aussi des soutiens de la part d'autres experts pour construire l'étalonnage requis.

\section{Une traduction juridique qui a choisi ses experts en écologie et en économie}

La DCE définit les conditions de référence d'un système écologique comme celui prévalant en l'absence ou la quasi-absence de perturbations anthropiques ${ }^{5}$. Cela correspond à des caractéristiques hydromorphologiques, physicochimiques et biologiques «non perturbées ", des concentrations en polluants de synthèse proches de zéro et des teneurs relevant du «bruit de fond » en polluants non synthétiques. La chimie de l'eau et la toxicologie permettent d'étalonner les concentrations en fonction des risques sanitaires associés. Du côté de l'écologie, l'étalonnage repose sur une typologie régionalisée des milieux aquatiques qui rend compte de la variation de biodiversité induite par les caractéristiques écorégionales (facteurs hydroclimatiques, habitat physique, facteurs trophiques et biotiques) sur des cours d'eau non perturbés de taille relativement similaire (Hughes et Larsen, 1988 ; Omernik, 1987). Le bon état correspond à un écart à cette référence n'entraînant pas de distorsion notable des biocénoses. Un écart plus notable est interprété comme le signe d'une perturbation des facteurs-clés déterminant la biodiversité localement. Cette approche suppose un retour possible au bon état en cas de suppression de la perturbation, sous réserve que cette dernière n'ait pas engendré d'irréversibilité.

Cette définition est proche de celle employée aux États-Unis dès 1972 dans le Clean Water Act (U.S. House of Representatives, 1972). Elle se réfère au caractère

\footnotetext{
5 Les expressions «perturbé »/ « non perturbé »/ « perturbations » sont utilisées 107 fois dans l'annexe $\mathrm{V}$ de la directive sans que la notion de perturbation y soit définie.
}

«naturel » de l'écosystème (dans ses structures et ses fonctions) et a un lien direct avec la notion d'intégrité biotique (Karr, 1981). Récemment, le concept et les critères permettant d'évaluer une déviation par rapport à cet état ont été codifiés aux États-Unis (Davies et Jackson, 2006) et en Australie (Water Reform Framework, Stoddard et al., 2006). La description de cet état de référence est liée à la connaissance de sites présentant les caractéristiques recherchées (conditions pristine, c'est-à-dire originelles), mais aussi, si besoin, à des éléments de connaissances historiques, voire d'expertise et/ou à des méthodes de modélisation simple.

La notion de conditions de référence est utilisée de longue date dans d'autres domaines que l'hydrobiologie, en particulier en écologie forestière (Swetnam et al., 1999). Des approches interdisciplinaires permettent de décrire les tendances évolutives des écosystèmes et/ou des paysages sur le long terme (millénaire). Ces reconstitutions alimentent un nouveau champ disciplinaire (Applied Historical Ecology) faisant explicitement appel à la notion de conditions de référence pour orienter la gestion des systèmes et les opérations de restauration (Egan et Howell, 2001).

Lorsque l'on s'intéresse à l'histoire de la rédaction de la DCE et des différentes influences ayant opéré, on peut voir que ce concept ne s'est que progressivement imposé. Les rédacteurs de la DCE étaient des fonctionnaires ou des contractuels de la Direction générale de l'environnement de la Commission européenne (DG environnement) relativement peu nombreux. Ils ont pu être identifiés et interrogés (Loupsans et Gramaglia, 2011). Il s'agit d'experts qui ont souvent travaillé pour d'autres services de la Commission ou dans des services environnementaux de pays membres. Ils s'inscrivent dans les préoccupations gestionnaires que nous avons évoquées en première partie. Cependant, ils se sont entourés de scientifiques écologues pour rédiger une première ébauche de la directive (Commission of the European Communities, 1994). Celle-ci ne fixait pas d'obligation de résultat et intégrait des considérations esthétiques et patrimoniales dans les objectifs de qualité. L'accent était mis sur la qualité de l'eau et secondairement sur la connectivité biologique au sein des réseaux hydrographiques comme le montre le recours à la notion d'ecological water quality. L'expression "reference conditions » n'était pas employée. Le texte décrit la bonne qualité écologique de l'eau et des sédiments comme la qualité adaptée à l'ensemble des besoins de l'écosystème, en considérant en particulier la nécessité de maintenir ses capacités autoépuratrices, un bon niveau de diversité biologique (plantes aquatiques, invertébrés, poissons, amphibiens, mammifères, oiseaux) et l'absence de toxiques et de maladies liées à des activités humaines. Chaque État membre restait maître de sa propre définition du bon état et de la mise en œuvre des mesures de 
restauration. On perçoit dans le texte une priorité donnée à des outils d'évaluation privilégiant le fonctionnement des hydrosystèmes plus que leur description.

Cette première proposition a été abandonnée parce qu'à l'époque le Conseil des ministres ne souhaitait pas s'imposer de nouvelles normes coûteuses en matière d'environnement. La seconde ébauche reprise en 1999 a été développée du point de vue juridique, mais ses concepts écologiques et économiques ont été simplifiés. Loupsans et Gramaglia (2011) ont montré que ses rédacteurs se sont appuyés sur un comité plus restreint, composé d'écologues plus enclins à définir le bon état selon la communauté biologique observée en l'absence ou quasi-absence de pressions anthropiques que sur des critères fonctionnels. Ces deux écoles de pensée en écologie correspondent à deux conférences internationales organisées sous l'égide de la DG environnement sur l'évaluation de la qualité des eaux, l'une en 1991 à Bruxelles qui mit en avant l'approche fonctionnelle (Newman et al., 1992), l'autre en 1998 à Vienne qui portait sur les méthodes d'évaluation de l'intégrité écologique des eaux courantes (Jungwirth et al., 2000 ; Kern, 1992). Cette dernière semble avoir influencé la seconde version de la directive. L'annexe $\mathrm{V}$ de la DCE assoit ainsi l'évaluation sur un écart à des conditions de référence stables ${ }^{7}$ définies sur des critères d'abondance et de richesse spécifique, associé à une régionalisation de l'environnement. Dans cette seconde version, les indicateurs retenus ont été restreints au milieu aquatique, au détriment $d u$ sédiment, de la flore et de la faune caractérisant le corridor fluvial dans son ensemble. Les altérations hydromorphologiques sont davantage prises en compte que dans la version antérieure. Du point de vue socioéconomique, la seconde version a abandonné les considérations patrimoniales et esthétiques. Ce partage des tâches entre l'écologie chargée de déterminer les normes environnementales et l'économie chargée d'optimiser des régulations, en jouant sur les prix une fois que les normes sont fixées, correspond à l'école néoclassique de l'économie de l'environnement (Anderson et Leal, 1991) ${ }^{8}$.

La production de normes en écologie pose la question de leur légitimité sociale. Pourquoi certains

\footnotetext{
6 L'intégrité biotique est une démarche à l'origine américaine et reprise dès la fin des années 1980 en Allemagne et en Autriche. Elle repose sur une vision ou Leitbild de l'état à atteindre pour un tronçon de cours d'eau défini principalement en référence aux structures floro-faunistiques datant d'avant l'industrialisation.

7 Les rédacteurs de la DCE avaient conscience d'une éventuelle dynamique (spontanée ou anthropogénique) de cet état écologique ( $c f$. note $\left.\mathrm{n}^{\circ} 11\right)$. Mais, dans leur esprit, cette dynamique était lente et ne remettait pas en cause l'objectif de restauration.

8 Les auteurs remercient Tina Rambonilaza pour cette précision d'ordre économique.
}

compartiments, certaines espèces ou certaines fonctions seraient-ils à préserver et d'autres pas ? Historiquement, les indicateurs écologiques ont été construits pour quantifier des problèmes rendus publics par des mobilisations sociales ou des communautés scientifiques à certaines échelles et à certaines époques. Leur légitimité à d'autres échelles et en d'autres temps n'est pas automatique. Or cette définition du bon état devait permettre de standardiser les différentes méthodes d'évaluation nationales afin de rendre le bon état comparable entre les États membres (processus d'intercalibration européenne). Dans la pratique, la mise en œuvre de ces principes s'est avérée extrêmement variable selon les États, en fonction des données et ressources disponibles, des bio-indicateurs et des types de masses d'eau considérés, voire en fonction d'écoles de pensées chez les hydrobiologistes et les gestionnaires. Les conditions de référence sont alternativement traduites par (Stoddard et al., 2006) :

- l'intégrité biotique ou l'état « naturel » pour lequel la variabilité sur le long terme est acceptée mais dans des limites relativement étroites (état stable) ;

- un état correspondant aux conditions rencontrées dans les milieux très peu perturbés. On accepte ici un minimum de perturbations notamment celles agissant sur de grands espaces ;

- un état historique antérieur à toute modification humaine considérée comme "importante» par les auteurs. Par exemple, ces derniers (Wallin et al., 2003) retiennent la période antérieure au développement de l'agriculture intensive et/ou de l'industrialisation (vers 1850 en Angleterre, dès 1750 en Allemagne) sans tenir compte des modifications antérieures ${ }^{9}$;

- l'état le moins perturbé pour un espace donné. Des critères sont définis pour sélectionner les sites (qualité de l'eau, aménagements, occupation du sol) ;

- le meilleur état potentiellement accessible. C'est l'état que l'on peut espérer obtenir sur un site relativement dégradé si on y applique toutes les mesures de gestion possibles en matière de restauration écologique (cas par exemple aux Pays-Bas).

Cette diversité d'approches reflète des points de vue assez contrastés parmi les experts écologues et scientifiques qui ont été associés à la traduction de la DCE en termes opérationnels. L'approche la plus commune en vigueur (dont celle de la France) est la sélection des états peu à pas perturbés sur la base d'une description des pressions anthropiques. L'Allemagne et l'Autriche se réfèrent plus strictement à la notion d'intégrité biotique, en combinant des approches historiques et expertes (Schmutz et al., 2000).

\footnotetext{
9 Ces approches négligent notamment les effets de l'agriculture itinérante sur brûlis ou des défrichements médiévaux sur les hydrosystèmes.
} 
Il est intéressant de noter que ce difficile étalonnage n'est pas un cas isolé des politiques européennes. R. Salais qui s'est intéressé à la mise en œuvre de la stratégie de Lisbonne sur les questions de l'emploi a pointé l'absence de définition commune d'un chômeur et d'un emploi (Salais, 2004). I. Bruno qui étudie la politique d'égalité des chances entre hommes et femmes et les modes de financement des retraites note également dans ces domaines des définitions communes très floues (Bruno et al., 2006).

\section{Les arguments en faveur d'une conception écologique plus dynamique}

Il semble que la DCE ait été largement inspirée par le paradigme d'écosystème, une vision a priori de la nature centrée sur les espèces (Clements, 1905 ; Elton, 1930 ; Tansley, 1935) et surtout de la notion de climax qui lui a été rapidement associée (Clements, 1936). Chaque unité écologique est considérée comme relativement constante face à la variabilité environnementale et à même de s'autoréguler tel un organisme (Balance of Nature Concept). Elle passe par différentes phases pour atteindre un état climacique et $s^{\prime} y$ maintient jusqu'à modifications majeures de l'environnement global (sur un pas de temps long). Les écosystèmes représentent ainsi des structures prédictibles, même si les cycles de perturbation sont des parties intégrantes de leur fonctionnement (retour à un état antérieur «optimal»). Odum (1953) et Whittaker (1975) populariseront le concept de climax et sa vision holistique associée. La notion de conditions de référence se rattache à cette vision privilégiant un état stable. Ce concept de climax a été remis en cause durant les dernières décennies à plusieurs titres et le caractère stable et prédictible des écosystèmes a été contesté ( $\mathrm{O}^{\prime}$ Neill, 2001).

Dans un premier temps, la notion de résilience a pris en compte la variabilité du système autour d'un état stable en réponse à des perturbations faibles, en termes de temps de retour (Pimm, 1984), mais surtout d'intensité maximale de perturbation supportable sans altération notable des fonctions (Holling, 1973). Plus récemment, et face à des échecs répétés de restauration de milieux naturels, c'est-à-dire la mise en évidence du caractère irréversible de la plupart des modifications subies, Scheffer et al. (2001) ont avancé le concept d'Alternative State Theory. Les écosystèmes, même lorsqu'ils subissent des modifications continues sur un gradient environnemental donné (naturel ou non), changent radicalement à partir d'un certain point (processus non linéaire) et ne sont pas à même de retourner à leur état initial lorsque l'on rétablit les conditions environnementales antérieures. Pour un même type d'écosystème, on peut donc avoir différents domaines de stabilité. On envisage ici clairement une composante "chaotique » dans la trajectoire des écosystèmes.

Une autre remise en question majeure de la notion de conditions de référence provient du constat que les modifications de son environnement par l'homme sont en fait significatives depuis plusieurs millénaires: la notion d'état non perturbé n'a plus de signification écologique $^{10}$. En reprenant l'idée d'écosystème et en l'élargissant, le concept de sociosystème (Fischer-Kowalski, 1997 ; Lapierre, 1992) reconnaît le caractère indissociable des caractéristiques naturelles et sociales de ces systèmes, et le rôle central des interactions homme-nature. Dans le champ de l'histoire environnementale et plus précisément de l'écologie historique, les auteurs partent du postulat d'une coévolution de l'homme et de la nature sur le long terme (depuis le Néolithique) de type non déterministe, mais dialectique. Les activités humaines ne sont pas systématiquement perçues comme synonymes de destruction, elles sont aussi source de création de paysages (Balée, 1998).

La distinction entre un état naturel et un état modifié par l'homme est trompeuse. Elle suppose que l'on puisse séparer ce qui relève d'une action de l'homme et ce qui relève de la dynamique propre d'un système naturel. Cela est relativement possible pour des pressions locales, à condition toutefois que des acteurs se soient mobilisés pour les rendre visibles publiquement et mesurables avec des indicateurs d'état écologique (Bouleau, 2006; Bouleau et al., 2009). Mais dans le cas des cours d'eau en permanence traversés par des flux solides, liquides et chimiques en provenance de l'amont, les transformations immémoriales des bassins versants par l'homme ( $\mathrm{y}$ compris la nature des couverts forestiers) rendent illusoire la définition d'un état non impacté. Le cas de la Drôme, récemment étudié, montre bien la succession temporelle des interactions entre occupation du bassin versant et dynamique fluviale (Pont et al., 2009). Il y a peu, Gunderson et Holling (2002) ont proposé la notion de panarchie pour rendre compte de la dynamique complexe de l'ensemble. Dans la même voie, Lévêque et van der Leeuw (2003) avancent le terme d'anthroposystème. Les dynamiques des systèmes écologiques ne peuvent plus être considérées comme obéissant aux seules lois bio-physico-chimiques et la distinction entre paramètres naturels et pressions anthropiques est artificielle.

Steyaert et Ollivier (2007), dans une analyse critique de la DCE, concluent à l'influence prépondérante du courant de pensée en écologie se focalisant sur la notion de stabilité des écosystèmes et considérant systématiquement les activités humaines comme des facteurs de perturbation. Récemment, Dufour et Piégay (2009) ont proposé de remplacer la stratégie de restauration visant

\footnotetext{
${ }^{10}$ La jurisprudence communautaire lui donnera peut-être un sens juridique.
} 
à définir l'état écologique à atteindre par rapport à une référence "naturelle» idéale (reference-based strategy) telle que définie dans la notion de visionary Leitbild (Kern, 1992), par une stratégie définissant des objectifs prenant en compte dès le départ les services rendus par les écosystèmes.

\section{Une solution technique médiane : des indicateurs se basant sur des critères fonctionnels}

Les écologues aquatiques ont ainsi émis de nombreuses critiques, à la fois pour des raisons conceptuelles, pour l'importance laissée aux démarches expertes et pour l'absence de prise en compte de critères fonctionnels au niveau des indicateurs biologiques (Friberg et al., 2011). Les indicateurs prévus par la DCE ne sont pas considérés comme des mesures convenables pour juger l'efficience de la restauration, mais les données qu'ils produisent ont néanmoins une certaine utilité. Ainsi, et bien que conscients des limites de l'approche adoptée dans la DCE, un grand nombre de scientifiques européens dressent un bilan globalement positif des dix premières années d'application de la directive (Hering et al., 2010).

L'obligation de recourir à des évaluations basées sur la qualité des biocénoses a amené à la mise en place de réseaux de suivis biologiques à l'aide de techniques d'échantillonnage mieux standardisées. Le recours à un nombre restreint d'indicateurs biologiques (et chimiques) a l'avantage de focaliser les efforts sur des objectifs techniques précis et communs permettant l'amélioration de ces méthodes. La nécessité d'harmonisation entre les pays européens (processus d'intercalibration) a permis un développement sans précédent des échanges internationaux et des travaux (plus de 1100 publications en relation avec la DCE en dix ans sur Web of Science, juillet 2009). Par son pragmatisme, la démarche adoptée a pu être appliquée dans la plupart des pays et, malgré toutes ses faiblesses, a largement amélioré le degré de comparabilité entre la qualité écologique des cours d'eau européens.

Comme la mise en œuvre de la DCE actuellement porte sur l'évaluation de l'état écologique des cours d'eau et sur des propositions d'action, les débats conceptuels sur les conditions de référence n'empêchent pas son opérationnalisation. Les démarches sont le plus souvent pragmatiques et cherchent à « identifier » les conditions de référence par l'examen des sites peu perturbés, sur la base de l'examen des pressions et sans réellement s'attacher à définir au préalable une vision théorique et commune. Le bilan serait beaucoup plus réservé dans le cas des milieux lacustres et des eaux de transition (estuaires, milieux marins côtiers) où la plus grande rareté des données biologiques disponibles et l'absence fréquente de développement d'indicateurs antérieurement à la DCE n'a pas encore permis une réelle harmonisation des évaluations.

Il faut également noter qu'un nombre de plus en plus grand de bio-indicateurs DCE-compatibles ne se basent plus seulement sur les listes floro-faunistiques, mais sur des critères fonctionnels en recourant à des traits écologiques et/ou biologiques. C'est en particulier le cas en France (Launois et al., 2011 ; Mondy et al., 2012 ; Pont et al., 2006). Par exemple, l'omnivorie est utilisée comme un indicateur de dégradation, alors que la spécialisation dans les habitats de reproduction rend compte d'une altération physique faible du milieu. Ces approches, couplées avec le recours à des méthodes de modélisation, s'avèrent efficaces pour construire des outils applicables à l'échelle européenne, facilitant ainsi les comparaisons entre systèmes (Pont et al., 2006).

\section{Évaluation écologique et changement climatique}

C'est du point de vue conceptuel que le changement climatique met le plus en lumière la contradiction entre le concept de conditions de référence et la nature dynamique et peu prédictible des trajectoires des écosystèmes aquatiques. Les implications possibles du changement climatique sur la mise en œuvre de la DCE ont fait l'objet d'un document d'orientation par un groupe d'experts européens (n.s., 2009). Les auteurs mettent l'accent sur les modifications à attendre des régimes hydrologiques et thermiques et des implications sur l'ensemble des processus géochimiques. Il reste cependant difficile de distinguer entre les effets des facteurs climatiques et les impacts des autres modifications d'origine anthropique.

Les conséquences seront également importantes sur les distributions de très nombreuses espèces et en particulier les espèces cryophiles (ex : Salmonidés). L'accessibilité à des milieux refuges pendant les périodes de stress (étiages, pics thermiques) deviendra un facteur majeur de maintien de ces populations (Buisson et Grenouillet, 2009 ; Pont, 2006). Il s'ensuit que les biocénoses dites de référence seront modifiées.

Cependant, la mise en œuvre de la directive devant être achevée d'ici 2027, il n'est pas clairement démontré aujourd'hui que les modifications du signal climatique liées à des impacts anthropogéniques seront distinguables, dans ce délai, de la variabilité climatique naturelle et/ou de l'effet d'autres activités humaines. Dans un premier temps ( $1^{\mathrm{er}}$ plan de gestion, 2010-2015), les mesures prises doivent considérer les effets possibles à terme du changement climatique (capacité d'adaptation). Pour 
les plans suivants, les États doivent au minimum évaluer les conséquences du changement climatique sur les impacts des autres pressions anthropiques et sur les programmes de suivi pour bâtir des plans résilients. Il faudra privilégier dans les projets de restauration les mesures « sans regret », c'est-à-dire suffisamment fiables et robustes pour rester viables dans un contexte climatique changeant et ne risquant pas de contrarier des mesures d'adaptation à ce changement prises par ailleurs.

La distinction entre variabilité naturelle et impacts anthropiques dans le fonctionnement des systèmes va apparaître de plus en plus artificielle. La modification climatique ne changera pas simplement l'état écologique non perturbé, mais également les impacts des altérations humaines sur les biocénoses (eutrophisation, couplage des régimes thermiques et de l'hydrologie, etc.) et toutes les incertitudes associées à l'évaluation.

Un certain nombre de réponses techniques sont envisagées pour pallier au moins partiellement cette dérive attendue des conditions de référence :

- d'une part, l'établissement de réseaux de suivis à long terme de sites considérés comme peu à pas impactés au niveau local afin de pouvoir détecter les évolutions des écosystèmes et de leurs biocénoses ;

- d'autre part, la construction de bio-indicateurs permettant de modéliser les conditions de référence à attendre dans un nouveau contexte climatique (Pont et al., 2006; Pont et al., 2007). Cela explique l'intérêt pour des modèles construits à l'échelle européenne s'appuyant sur un champ d'observations incluant des gradients environnementaux les plus étendus possibles.

Les implications du changement climatique sur l'évaluation de l'état écologique vont donc soulever des problèmes, mais ceux-ci apparaissent relativement solubles en raison du cadre temporel limité de la DCE (20002027).

\section{L'agrégation des indicateurs dans la DCE : entre principe de précaution et logique économique}

D'un point de vue opérationnel, le principe d'agrégation des indicateurs dans la DCE présente finalement davantage de difficultés. Le modèle pression/impact, dont nous avons discuté les limites, est utilisé dans la directive comme base unitaire d'évaluation. Les indicateurs sont ensuite agrégés selon des règles qui réduisent la logique de compensation. L'annexe $\mathrm{V}$ de la DCE définit l'état de chaque masse d'eau de surface par la plus mauvaise note obtenue sur tous les indicateurs ${ }^{11}$. Cette

\footnotetext{
${ }^{11}$ Les Anglo-Saxons résument cette règle par la formule « one out, all out ».
}

règle n'a pas de fondement en écologie parce que la dégradation d'un paramètre n'est pas forcément significative. Elle se comprend davantage comme un principe de gestion visant à prévenir les stratégies de compensation d'un paramètre par un autre. Il s'agirait d'éviter toute dégradation dans un contexte où il n'est pas possible a priori de pondérer l'importance des différents indicateurs. On peut y reconnaître un principe de précaution dont on sait qu'il est très prégnant au sein de la DG environnement (Cini, 1996).

Les rédacteurs pensaient en outre que les indicateurs biologiques pointeraient les causes des dégradations, de sorte qu'une mauvaise note serait un outil pour agir. Mais ce raisonnement supposait une relation bijective entre une pression et un ensemble d'indicateurs, ce qui ne s'est pas révélé exact pour deux principales raisons.

En premier lieu, les experts ont surestimé les capacités des indicateurs biotiques à répondre de manière spécifique aux différents types de pression. Sauf quelques cas particuliers comme la réponse phytoplanctonique à un accroissement des teneurs en phosphore, les indicateurs répondent en général à un cocktail de pressions. Les résultats du processus d'intercalibration européenne ont montré que les relations de sensibilité des bioindicateurs aux principaux types de pression, bien que significatives, n'étaient pas aussi strictes que le laissaient entendre les prises de positions antérieures des experts nationaux (Pont, 2011). Cela est lié d'une part à l'existence d'incertitudes importantes associées aux évaluations (erreurs d'échantillonnage en particulier), et d'autre part au principe même des bio-indicateurs qui sont utilisés pour leur capacité d'intégrer des perturbations d'origines diverses.

De plus, dans un très grand nombre de cours d'eau, et en particulier dans les zones aval, les pressions sont le plus souvent multiples, associant à la fois la dégradation de la qualité de l'eau et la modification des lits fluviaux et des écoulements. C'est ainsi le cas de toutes les zones urbanisées et/ou industrialisées. Mais cela concerne également les zones d'agriculture intensive (rectification des cours d'eau associée aux pollutions diffuses).

Dans ce contexte, la règle de la plus mauvaise note présente de sérieuses contraintes. Tous les indicateurs écologiques sont d'abord sensibles à la qualité de l'eau et, de façon secondaire, aux altérations physiques du milieu (Marzin et al., 2012). Seuls peut-être les poissons, $\mathrm{du}$ fait des migrations des espèces diadromes présentent une réponse spécifique à l'altération de la connectivité longitudinale. Borja et Rodriguez (2010), puis van de Bund et Caroni (2012) ont montré que cette règle tendait à déclasser les masses d'eau lorsque les incertitudes associées aux indicateurs étaient fortes, que le nombre $\mathrm{d}$ 'indicateurs augmentait et que ces derniers étaient redondants entre eux. 
Ainsi les règles d'agrégation des critères n'empêchent pas la substitution des pressions, mais déclassent les eaux évaluées selon le plus grand nombre de critères.

\section{De l'évaluation à la restauration}

La question des conditions de référence réapparaît au premier plan lorsqu'on passe de l'évaluation à la restauration qui est l'objectif premier des plans de gestion (2015-2021 puis 2021-2027) prévus par la DCE.

L'un des principes guidant les opérations de restauration est que le rétablissement des conditions abiotiques antérieures et des régimes de perturbations associés entraîne un rétablissement des communautés biologiques prévalant auparavant. On fait appel implicitement au principe de succession des communautés (Successional-Based Management Effort). Mais, en fait, les résultats des restaurations sont souvent inattendus: le système ne retourne pas à son état antérieur (Suding et al., 2004). Ce résultat peut être expliqué par l'absence actuelle de systèmes permettant de définir une référence, un changement des conditions à des échelles supra-locales (paysage, bassin versant, climat), une modification irréversible de certains paramètres environnementaux, des modifications de la connectivité à l'échelle des bassins, la modification du cortège d'espèces, de nouvelles interactions biotiques et l'altération des feedbacks biotiques-abiotiques entraînant une modification durable des conditions bio-géochimiques (Henri et Amoros, 1995 ; Nilsson et al., 2007 ; Suding et al., 2004). Moss (2008) insiste également sur le temps de réponse qui est parfois très long et rend difficile l'évaluation des interventions. Sur ces bases, Hughes et al. (2005) concluent à l'impossibilité de définir des objectifs trop précis en matière de restauration.

Quand on s'intéresse à un seul type de "pression humaine » facilement identifiable (eutrophisation, pollution organique), la situation reste relativement aisée et correspond au plus grand nombre des interventions menées lors des décennies antérieures. En revanche, si l'on cherche à corriger de multiples interventions / modifications anthropiques sur le milieu souvent anciennes (ex : cours d'eau), la question de la définition des objectifs de restauration et donc de leur adéquation par rapport aux « conditions de référence » est complexe.

Dans plusieurs pays cependant, la crainte d'être évalué sur des critères peu contrôlables génère des stratégies visant à minimiser la détection des situations problématiques. Nous avons pu observer au niveau local des déplacements de points de mesure hors des zones les plus perturbées et au niveau national la tentation d'éliminer des métriques jugées trop sensibles. Lors d'entretiens, des experts locaux nous ont rapporté des cas où les données ainsi produites ne permettent plus de repérer des perturbations pour lesquelles des acteurs sociaux s'étaient mobilisés sur le terrain.

\section{Bilan et conclusion}

En définitive, la mise en place d'une directive prenant directement en compte les biocénoses dans l'évaluation de l'état, sans se limiter à l'examen de la qualité physicochimique des eaux, a permis une réelle avancée du point de vue environnemental, mais a cependant posé des problèmes sérieux, pour certains relativement nouveaux, et pas tous surmontés douze ans après la publication et la mise en œuvre du texte.

La DCE est héritière d'une nouvelle forme de gouvernement qui s'appuie sur l'évaluation par indicateurs. Celle-là exige une définition quantitative des objectifs pour pouvoir comparer économiquement différentes actions publiques. Les experts qui ont opéré la traduction des objectifs écologiques en indicateurs quantitatifs ont le plus souvent utilisé un corpus théorique minoritaire en décalage avec les consensus actuels en écologie. Ces derniers se sont éloignés progressivement de la notion de systèmes en équilibre et prévisibles pour adopter de plus en plus une vision dynamique, dans laquelle la trajectoire des écosystèmes inclut des aspects aléatoires la rendant de moins en moins prévisible. De ce point de vue, évoquer des conditions stables de référence relève d'une conception des écosystèmes que l'on peut qualifier de "climacique», qui s'oppose à une conception plus adaptative où les perturbations passées tendent à influer de façon irréversible leurs trajectoires et où l'homme est partie prenante de cette dynamique.

Il faut noter cependant que la relative stabilité d'un écosystème au cours d'une période limitée est une référence qui reste utile dans bien des cas pour mesurer des dégradations. C'est dans ce contexte que la notion de référence a connu un certain succès. Bien qu'évoquée au singulier, cette notion a été déclinée de manière variée, en ajustant la période d'origine, la fréquence de mesure et les lieux d'échantillonnage de manière à caractériser un impact écologique particulier qui posait un problème social.

La DCE a permis l'établissement de réseaux de mesures chimiques et biologiques homogènes sur toutes les eaux alors que beaucoup n'étaient pas surveillées auparavant. Le processus d'intercalibration des différentes méthodes d'évaluation a poussé la plupart des États membres à définir a minima des conditions de référence communes et à les identifier sur la base des réseaux de sites peu perturbés, soit une démarche pragmatique. Les données produites par ces réseaux seront précieuses pour comprendre le fonctionnement écologique des 
milieux aquatiques. Pour cela, les stratégies de dissimulation des situations problématiques devront rester minoritaires et ces réseaux devront intégrer de plus en plus la nouvelle génération de bio-indicateurs, basée sur des caractéristiques fonctionnelles des biocénoses, qui est mieux à même de permettre une analyse comparative des masses d'eau à l'échelle européenne.

En ce qui concerne les impacts du changement climatique sur l'évaluation de l'état écologique, il est clair que cette variabilité macro-environnementale additionnelle met encore plus à mal le concept de conditions de référence. Cependant, le cadre temporel limité de la DCE (2000-2027) autorise des approches permettant de réviser les conditions de références locales pour prendre en compte le nouvel environnement régional (réseaux de suivis, modélisation des conditions de référence) ${ }^{12}$.

En revanche, les implications les plus importantes concerneront la restauration des milieux, car ces démarches se placent sur des échelles de temps plus longues que celle de l'application de la directive. La définition d'objectifs de restauration et leur adéquation par rapport aux conditions de référence est déjà une question ouverte. En outre, le bon état défini par la DCE agrège plusieurs critères en une référence unique. Or les critères écologiques reflètent des préférences pour certaines espèces, certains paysages ou certaines fonctions qui sont elles-mêmes héritières de mobilisations sociales situées dans le temps et dans l'espace (Bouleau, 2007 ; Depraz, 2007 ; Le Floch, 2005) ${ }^{13}$. L'agrégation théorique de ces critères sur chaque masse d'eau donne la priorité à la plus mauvaise note sans souci de cohérence territoriale ou politique. Cette agrégation n'a pas forcément non plus de cohérence du point de vue écologique. Alors que le New Public Management dont est inspiré la DCE exige une mesure convenable de l'efficience, les bio-indicateurs de la DCE ne remplissent pas ce rôle aujourd'hui. La nécessité de privilégier à l'avenir des mesures «sans regret » et compatibles avec les mesures d'adaptation prises par ailleurs, ainsi que les orientations privilégiant le recours aux énergies renouvelables, va nécessiter une redéfinition des méthodologies.

\footnotetext{
${ }^{12}$ L'annexe V (1.3.1) prévoit que « les États membres établissent des programmes de contrôle de surveillance afin de fournir des informations pour [...] évaluer les changements à long terme des conditions naturelles ».

13 Pour les écosystèmes aquatiques, G. Bouleau a étudié comment l'intérêt porté aux invertébrés benthiques en France est né dans des communautés scientifiques proches de la pêche sportive en eau douce. Concernant les écosystèmes terrestres, $\mathrm{S}$. Le Floch a retracé la construction sociale d'une préférence pour des espaces « ouverts » et $S$. Depraz a relaté l'émergence récente d'un intérêt pour des fonctions non productives de la nature dans les paysages ruraux des nouveaux Länder.
}

Une des pistes possibles pour le futur pourrait être de travailler sur les fonctions écologiques et leur articulation avec une meilleure prise en compte des biens et services rendus par les écosystèmes (Dufour et Piégay, 2009). Cependant, en cherchant à associer aux fonctions écologiques des services rendus par les écosystèmes, y compris des services culturels, on ne retient que la valeur instrumentale de l'environnement. Or l'environnement est aussi le réceptacle de valeurs morales, d'identités et d'attachements qui ne peuvent pas être traduits en termes de bénéfices incontestés. Étant donné les incertitudes sur la restauration, la préservation de l'environnement, là où il existe et où il a coévolué avec la société, reste d'actualité.

Le croisement de nos regards d'écologue et de sociologue sur la notion de référence met en lumière la dimension historique des trajectoires humaines et naturelles, qu'on les étudie séparément ou bien en tenant compte de leurs interactions. Les situations présentes dépendent d'héritages passés et leur histoire ne se raconte pas de la même façon selon l'échelle spatiale et l'origine temporelle considérées. Une question de recherche intéressante pour l'avenir est de voir comment les différents groupes sociaux se saisissent ou non de la DCE et de son outillage (réseaux de mesures, expertise, concepts, documents de programmation, étapes de décision) pour orienter l'avenir de leur environnement dans un contexte changeant.

\section{Références}

[n.s.], 2009. Common implementation strategy for the Water Framework Directive (2000/60/EC). Guidance document No. 24, River basin management in a changing climate, European communities (online: http://www.sednet.org/download/ directive_climate_adaptation_management_climate.pdf).

Anderson, T., Leal, L., 1991. Free Market Environmentalism, Boulder (CO), Westview Press.

Balée, W., 1998. Advances in Historical Ecology, New York, Columbia University Press.

Bezes, P., 2005. Le renouveau du contrôle des bureaucraties: l'impact du New Public Management, Informations Sociales, $126,26-37$.

Borja, A., Rodríguez, J.G., 2010. Problems associated with the "one-out, all-out" principle, when using multiple ecosystem components in assessing the ecological status of marine waters, Marine Pollution Bulletin, 60, 8, 1143-1146.

Bouleau, G., 2006. Le débat sur la qualité de l'eau : comment des données peuvent devenir des indicateurs ?, Ingénieries EAT, 47, 29-36.

Bouleau, G., 2007. La gestion française des rivières et ses indicateurs à l'épreuve de la directive cadre. Thèse de doctorat, AgroParisTech, Engref, Paris.

Bouleau, G., Argillier, C., Souchon, Y., Barthélémy, C., Babut, M., 2009. How ecological indicators construction reveals social changes: The case of lakes and rivers in France, Ecological Indicators, 9, 6, 1198-1205. 
Bruno, I., Jacquot, S., Mandin, L., 2006. Europeanization through its instrumentation: Benchmarking, mainstreaming and the open method of co-ordination... Toolbox or Pandora's box?, Journal of European Public Policy, 13, 4, 519-536.

Buisson, L., Grenouillet, G., 2009. Contrasted impacts of climate change on stream fish assemblages along an environmental gradient, Diversity and Distributions, 15, 4, 613-626.

Cini, M., 1996. La Commission européenne : lieu d'émergence de cultures administratives. L'exemple de la DG IV et de la DG XI, Revue Française de Science Politique, 46, 3,457-473.

Clements, F.E., 1905. Research Methods in Ecology, Lincoln (NE), University Printing.

Clements, F.E., 1936. Nature and structure of the climax, Journal of Ecology, 24, 1, 252-284.

Collins, K., Earnshaw, D., 1992. The implementation and enforcement of European community environment legislation, Environmental Politics, 1, 4, 213-249.

Commission of the European Communities, 1994. Proposal for a Council Directive on the ecological quality of water. COM (93), 94/0152 SYN, Brussels (online : http:/ / eur-lex.europa.eu/ LexUriServ/LexUriServ.do?uri=OJ:C:1994:222:FULL:EN: PDF).

Davies, S.P., Jackson, S.K., 2006. The biological condition gradient: A descriptive model for interpreting change in aquatic ecosystems, Ecological Applications, 16, 4, 1251-1266.

Dehousse, R., 2004. La méthode ouverte de coordination: quand l'instrument tient lieu de politique, in Lascoumes, P., Galès, P.L. (Eds), Gouverner par les instruments, Paris, Presses de Sciences Po, 331-356.

Depraz, S., 2007. Campagnes et naturalité : la redéfinition d'un rapport à la nature dans les espaces ruraux des nouveaux Länder allemands, Revue d'Études Comparatives Est-Ouest, 38, 3, 35-152.

Douglas, M., 1999. Comment pensent les institutions, Paris, La découverte, Mauss.

Dufour, S., Piégay, H., 2009. From the myth of a lost paradise to targeted river restoration: Forget natural references and focus on human benefits, River Research and Applications, 25, 5, 568-581.

EEA, 1995. Inland Waters - Europe's Environment: The Dobris Assessment 1994 (chapter 5), European Environmental Agency (online: http://www.aughty.org/pdf/dobris.pdf).

Egan, D., Howell, E.A., 2001. The Historical Ecology Handbook: A Restorationist's Guide on Reference Ecosystems, Washington, Island Press.

Elton, C., 1930. Animal Ecology and Evolution, Oxford (UK), Clarendon Press.

Fernandez, S., Bouleau, G., Treyer, S., 2011. Reconsidérer la prospective sur l'eau en Europe dans ses dimensions politiques, Développement Durable et Territoires, 2, 3.

Fischer-Kowalski, M., 1997. On the development of concept and methodology of society's metabolism, Abteilung Soziale Ökologie (version anglaise), 57, Interuniversitäres Institut für interdisziplinäre Forschung und Fortbildung.

Friberg, N., Bonada, N., Bradley, D.C., Dunbar, M.J., Edwards, F.K., Grey, J., Hayes, R.B., Hildrew, A.G., Lamouroux, N., Trimmer, M., Woodward, G., 2011. Biomonitoring of human impacts in freshwater ecosystems: The good, the bad, and the ugly, Advances in Ecological Research, 44, 1-68.
Gunderson, L.H., Holling, C.S., 2002. Panarchy: Understanding Transformations in Human and Natural Systems, Washington, Island Press.

Henri, C.P., Amoros, C., 1995. Restoration ecology of riverine wetlands: I. A scientific base, Environmental Management, 19, 891-902.

Hering, D., Borja, A., Carstensen, J., Carvalho, L., Elliott, M., Feld, C.K., Heiskanen, A.-S., Johnson, R.K., Moe, J., Pont, D., Lyche Solheim, A., van de Bund, W., 2010. The European Water Framework Directive at the age of 10: A critical review of the achievements with recommendations for the future, Science of the Total Environment, 408, 19, 40074019.

Holling, C., 1973. Resilience and stability of ecological systems, Annual Review of Ecology, Evolution, and Systematics, 4, 1-23.

Hughes, F.M.R., Colston, A., Mountford, J.O., 2005. Restoring riparian ecosystems: The challenge of accommodating variability and designing restoration trajectories, Ecology and Society, 10, 1, 12.

Hughes, R.M., Larsen, D.P., 1988. Ecoregions : An approach to surface water protection, Journal Water Pollution Control Federation, 60, 486-493.

Jungwirth, M., Muha, S., Schmutz, S. (Eds), 2000. Assessing the Ecological Integrity of Running Waters. Proceedings of the International Conference held in Vienna, Austria, 9-11 November 1998 (reprinted from Hydrobiologia, 422-423), Dordrecht, Kluwer Academic Press.

Kaika, M., Page, B., 2003. The EU Water Framework Directive: European policy making and the changing topography of lobbying (part 1), European Environment, 13, 6, 314-327.

Karr, J.R., 1981. Assessment of biotic integrity using fish communities, Fisheries, 6, 6, 21-27.

Kern, K., 1992. Rehabilitation of streams in South-West Germany, in Boon, P.J., Calow, P., Petts, G.E. (Eds), River Conservation and Management, Chichester (UK), John Wiley \& Sons Ltd, 321-336.

Lapierre, J.-W., 1992. L'Analyse des systèmes : l'application aux sciences sociales, Paris, Syros.

Launois, L., Veslot, J., Irz, P., Argillier, C., 2011. Selecting fishbased metrics responding to human pressures in French natural lakes and reservoirs: Towards the development of a fish-based index (FBI) for French lakes, Ecology of Freshwater Fish, 20, 1, 120-132.

Le Floch, S., 2005. La «fermeture du paysage » : au-delà du phénomène, petite chronique d'une construction sociale, L'Espace Géographique, 34, 1, 49-64.

Lévêque, C., van der Leeuw, S. (Eds), 2003. Quelle nature voulons-nous? Pour une approche socio-écologique du champ de l'environnement, Paris, Elsevier.

Loupsans, D., Gramaglia, C., 2011. L'expertise sous tensions : cultures épistémiques et politiques à l'épreuve de l'écriture de la directive cadre européenne sur l'eau, L'Europe en Formation, Centre international de formation européenne, 3, 361, 87-114.

Majone, G., 1996. La Communauté européenne : un État régulateur, Paris, Montchrestien.

Marzin, A., Archaimbault, V., Belliard, J., Chauvin, C., Delmas, F., Pont, D., 2012. Ecological assessment of running waters: Do macrophytes, macroinvertebrates, diatoms and fish 
show similar responses to human pressures?, Ecological Indicators, 23, 56-65.

Mondy, C.P., Villeneuve, B., Archaimbault, V., UsseglioPolatera, P., 2012. A new macroinvertebrate-based multimetric index (I2M2) to evaluate ecological quality of French wadeable streams fulfilling the WFD demands: A taxonomical and trait approach, Ecological Indicators, 18, 452-467.

Moss, B., 2008. The Water Framework Directive: Total environment or political compromise?, Science of the Total Environment, 400, 1-3, 32-41.

Newman, P.J., Piavaux, M.A., Sweeting, R.A. (Eds), 1992. River water quality ecological assessment and control. Proceedings of the International Conference on River Quality-Ecological Assessment and Control, Brussels, 16 december 1991, Commission of the European Communities, Luxembourg, No.14606.

Nilsson, C., Jansson, R., Malmqvist, B., Naiman, R.J., 2007. Restoring riverine landscapes: The challenge of identifying priorities, reference states, and techniques, Ecology and Society, 12, 1 (online : http://www.ecologyandsociety.org/ vol12/iss1/art16/).

O'Neill, R.V., 2001. Is it time to bury the ecosystem concept? (with full military honors, of course!), Ecology, 82, 12, 32753284.

OCDE, 1994. Indicateurs d'environnement: Corps central de l'OCDE, Paris, OCDE.

OCDE, 1995. La gestion publique en mutation : les réformes dans les pays de l'OCDE, Paris, OCDE.

Odum, E.P., 1953. Fundamentals of Ecology, Philadelphia (PA), W. B. Saunders.

OECD, 1993. Organisation for Economic Co-operation and Development (OECD) core set of indicators for environmental performance reviews, Paris, OECD Environment Monographs No. 83.

Omernik, J.M., 1987. Ecoregions of the Conterminous United States, Annals of the Association of American Geographers, 77, 1, 118-125.

Perrin, B., 2006. Moving from Outputs to Outcomes: Practical Advice from Governments Around the World, Washington, World Bank, IBM Center for the Business of Government.

Pimm, S.L., 1984. The complexity and stability of ecosystems, Nature, 307, 321-326.

Pont, D. (Ed.), 2006. Conséquences du changement global sur la distribution et la démographie du Chabot (Cottus gobio) : une approche intégrative, Paris, Institut français de la biodiversité, MEDD-GICC.

Pont, D. (Ed.), 2011. Water Framework Directive. Intercalibration Phase 2: River Fish European Intercalibration Group. Final Report to ECOSTAT, European Commission Directorate General Joint Research Centre.

Pont, D., Hugueny, B., Beier, U., Goffaux, D., Melcher, A., Noble, R., Rogers, C., Roset, N., Schmutz, S., 2006. Assessing river biotic condition at the continental scale: $\mathrm{A}$ European approach using functional metrics and fish assemblages, Journal of Applied Ecology, 43, 70-80.

Pont, D., Hugueny, B., Rogers, C., 2007. Development of a fishbased index for the assessment of "river health" in Europe: The European Fish Index (EFI), Fisheries Management and Ecology, 14, 427-439.
Pont, D., Piégay, H., Farinetti, A., Allain, S., Landon, N., Liébault, F., Dumont, B., Richard-Mazet, A., 2009. Conceptual framework and interdisciplinary approach for the sustainable management of gravel-bed rivers: The case of the Drôme River basin (S.E. France), Aquatic Ecology, 71, 356-370.

Richardson, J., 1994. EU water policy: Uncertain agendas, shifting networks and complex coalitions, Environmental Politics, 3, 4, 139-167.

Salais, R., 2004. La politique des indicateurs: du taux de chômage au taux d'emploi dans la stratégie européenne pour l'emploi (SEE), in Zimmermann, B. (Ed.), Action publique et sciences sociales, Paris, Maison des sciences de l'homme.

Scheffer, M., Carpenter, S., Foley, J.A., Folke, C., Walker, B., 2001. Catastrophic shifts in ecosystems, Nature, 413, 11, 591-596.

Schmutz, S., Kaufmann, M., Vogel, B., Jungwirth, M., Muhar, S., 2000. A multi-level concept for fish-based, river-typespecific assessment of ecological integrity, in Jungwirth, M., Muhar, S., Schmutz, S. (Eds), Assessing the Ecological Integrity of Running Waters, Dordrecht, Kluwer Academic Publishers, 279-289.

Steyaert, P., Ollivier, G., 2007. The European Water Framework Directive: How ecological assumptions frame technical and social change, Ecology and Society, 12, 1, 25 (online: http://www.ecologyandsociety.org/vol12/iss1/art25/).

Stoddard, J.L., Larsen, D.P., Hawkins, C.P., Johnson, R.K., Norris, R.H., 2006. Setting expectations for the ecological condition of streams: The concept of reference condition, Ecological Applications, 16, 4, 1267-1276.

Suding, K.N., Gross, K.L., Houseman, G.R., 2004. Alternative states and positive feedbacks in restoration ecology, Trends in Ecology and Evolution, 19, 1, 46-53.

Swetnam, T.W., Allen, C.D., Betancourt, J.L., 1999. Applied historical ecology: Using the past to manage the future, Ecological Applications, 9, 4, 1189-1206.

Tansley, A.G., 1935. The use and abuse of vegetational concepts and terms, Ecology, 16, 3, 284-307.

U.S. House of Representatives, 1972. Report of the Committee on Public Works, United States House of Representatives, with additional and supplemental views. H.R. 11896 to amend the Federal Water Pollution Control Act, $92^{\text {nd }}$ Congress, $2^{\text {nd }}$ session, House Report 92-911, U.S. Government Printing Office, Washington.

Van de Bund, W., Caroni, R., 2012. Combination of Biological Quality Elements towards complete water body assessment. Oral communication, WISER Final Conference "Current Questions in Water Management", Tallinn, Estonia, 25-26 January.

Wallin, M., Wiederholm, T., Johnson, R.K., 2003. Final guidance on establishing reference conditions and ecological status class boundaries for inland surface waters EU Common Implementation Strategy (CIS) for the Water Framework Directive, CIS Working Group 2.3, REFCOND (online: http:// www.minenv.gr/pinios/00/odhgia/7th_draft_refcond_ final.pdf).

Whittaker, R.H., 1975. Communities and Ecosystems, New York, Macmillan Publishing Co.

Accepté le 9 septembre 2013. 